\title{
Reliability of RT3 accelerometer for measuring mobility in people with multiple sclerosis: Pilot study
}

\author{
Leigh Hale, PhD; ${ }^{*}$ Kimberly Williams; Craig Ashton; Tim Connole; Hayley McDowell; Colleen Taylor \\ Centre for Physiotherapy Research, University of Otago, Dunedin, New Zealand
}

\begin{abstract}
This pilot study investigated the test-retest reliability of an RT3 accelerometer (RT3) for measuring motion in people with multiple sclerosis (MS). Ten people with MS (mean age 49 yr; Extended Disability Status Scale mean +/standard deviation $=3.4+/-1.3$ ) and ten nondisabled people (mean age $40 \mathrm{yr}$ ) wore the RT3 while they performed three discrete mobility tasks on two occasions separated by 1 week. The intraclass correlation coefficients (ICCs) calculated from the RT3 motion data for the group with MS were 0.64 for the 5 -minute walk test ( $p=0.01$ ), 0.50 for the timed up and go test $(p=0.05)$, and 0.76 for the stair-climbing task $(p=0.002)$. For the control group, these values were $0.65(p=0.01),-0.04(p=$ $0.54)$, and $0.39(p=0.11)$, respectively. We found that the RT3 can potentially provide stable data when measuring walking, but a more robust, yet participant-friendly, method of attaching the RT3 is required. Both participant groups demonstrated inconsistencies in motor-task performance, highlighting a potential source of measurement error that would need to be addressed when future studies are designed. Based on the results of the 5-minute walk test in this study, a sample of 53 participants would be required to obtain an ICC value with a $95 \%$ confidence interval of width 0.2 using two repeat measurements.
\end{abstract}

Key words: 5-minute walk test, accelerometer, mobility, multiple sclerosis, rehabilitation, reliability, RT3, stair-climbing task, test-retest reliability, timed up and go test.

\section{INTRODUCTION}

Multiple sclerosis (MS) is a chronic demyelinating disease that frequently results in reduced physical function and decreased levels of physical activity and fitness [1]. Although exercise programs for people with MS can increase fitness levels [2-4], whether these programs actually increase the amount of daily physical activity performed is not clear. This omission may be a result of the difficulties inherent in measuring physical activity in the free-living situation. Activity questionnaires and diaries are commonly used to measure physical activity levels at home. However, these methods rely on recall and honest reporting and require individuals to have no cognitive deficits and no potential for bias in reporting

\footnotetext{
Abbreviations: $\mathrm{au}=$ activity units, $\mathrm{CI}=$ confidence interval, EDSS $=$ Extended Disability Status Scale, ICC $=$ intraclass correlation coefficient, $\mathrm{MS}=$ multiple sclerosis, $\mathrm{MVM}=$ mean vector magnitude, RT3 = RT3 accelerometer, SEM = standard error of measurement.

*Address all correspondence to Leigh Hale, PhD; Centre for Physiotherapy Research, University of Otago, PO Box 56, Dunedin, New Zealand; +64-3-479-5425; fax: +64-3479-8414. Email: leigh.hale@otago.ac.nz

DOI: 10.1682/JRRD.2005.09.0155
} 
results [5-6]. ${ }^{*}$ Motion sensors, such as pedometers and accelerometers, provide an objective measure of physical activity. Pedometers are simple to use and inexpensive, but they have been found to be less accurate at slow walking speeds [7-8]. Triaxial accelerometers are electronic devices that measure acceleration along three anatomical axes, ${ }^{*}$ providing not only an indication of movement but also the intensity of the movement (http:// www.stayhealthy.com).

The RT3 accelerometer (RT3) (Stayhealthy, Inc, Monrovia, California) is a new, small ( size of a pager), triaxial monitor [9-14]. The integration of the three electronic measuring devices into a single computer chip purportedly increases the accuracy with which the RT3 measures motion and this accuracy, together with the lightness of the RT3 (weight $=65 \mathrm{~g}$ ), provides the model with an advantage over its predecessors [9]. The RT3 is capable of collecting and storing data in either 1-second epochs for 9 hours or 1-minute epochs for 21 days and has no external controls that could enable the person being monitored to manipulate or change the settings [9]. Thus, the RT3 is potentially suitable for measuring discrete movement tasks or for long-term tracking of physical activity in the home environment. Furthermore, accelerometer data (provided as "activity units" [au]) can estimate energy usage (in kilocalories). The RT3 software computes activity kilocalories from the integrated acceleration data collected by the RT3. The formula used to convert the raw data to activity kilocalories is proprietary and based on mass and integrated acceleration. It was developed through internal clinical trials [9].

Whereas a number of studies have examined the properties of the earlier triaxial accelerometer models in a number of population groups [1,5,15-21], few studies have investigated the latest, allegedly more sensitive and expedient model, the RT3 [9-14]. Four studies have validated the RT3. One study investigated the validity of the RT3 for measuring physical activity in boys and men [9]. The concurrent validity of the RT3 has been assessed against other accelerometer models [10-11] and against an activity questionnaire [12]. The RT3 has been reported to have high intermonitor reliability (intraclass correlation coefficient $[$ ICC $]=0.99, p<0.001$ ) when tested on a mechanical vibration table [13]. Testing reliability during human motion is more complex than with a mechanical

*Mulcare JA, Mathews T. Unpublished observations; 2004. device. Possible sources of measurement error on repeated testing include not only the unit's testing capabilities but also possible movement variations at attachment site and the inconsistencies of repeated human movements. In a subsequent study, the reliability of eight RT3s was investigated over two repeated trials of six activities performed by one nondisabled adult [14]. The RT3 monitors provided stable data over repeated activities. No significant intermonitor differences within activities were demonstrated for lower intensity activities (resting, walking at $4 \mathrm{~km} / \mathrm{h}$, sit-to-stand); however, variation between 21 and 82 percent was noted for higher intensity activities (walking at $6-10 \mathrm{~km} / \mathrm{h}$ ) [14].

To date, no studies have investigated the reliability of the RT3 for use with people with physical disabilities, either in the laboratory or free-living setting. This pilot study investigated the test-rest reliability of the RT3 for consistently measuring motion in a small sample of adults with MS and nondisabled adults in a laboratory setting. To test the hypothesis that the RT3 accelerometer would have good test-retest reliability in measuring motion, we asked 10 adults with MS and 10 nondisabled adults to perform three mobility tasks on two occasions separated by 1 week.

\section{METHODS}

\section{Participants}

Ten participants aged at least $18 \mathrm{yr}$ with a definite diagnosis of MS [22] and the ability to walk with or without assistive devices for $>5$ minutes were recruited from the local MS Society. Participants had to be in a remissive state of their disease process and have no disorder other than MS that would affect their mobility. Ten nondisabled adult volunteers were recruited from the staff of a large local organization as a control group. All participants read and signed the informed consent form approved by the local regional ethics committee.

\section{Pretest Assessments}

To characterize the MS participants, we recorded general information relating to participants' MS condition, medical history, and Extended Disability Status Scale (EDSS) [23]. MS participants' level of mobility was assessed with the Rivermead Mobility Index [24]. 


\section{Procedures}

Test-retest reliability of a single RT3 accelerometer was investigated on two occasions separated by 1 week in both MS and control participants. On each occasion, participants were tested at the same time of the day and were asked to perform the same three mobility tasks. All participants were asked to wear similar clothing and shoes on the two occasions. The temperature in the laboratory was controlled and standardized for both test occasions at $22{ }^{\circ} \mathrm{C}$.

\section{Equipment}

The single RT3 was programmed via computer interface with the participant's personal data (sex, age, height, and weight) before testing and set to sample data for all three axes every second. Westerterp recommended that motion monitors be placed as close to the body's center of gravity as possible [5]. The RT3 in this study was therefore worn positioned at the center of the back, attached to the participants' trousers or skirt belt via an attachable clip. Because calibrating an RT3 unit is not possible, the reliability of the unit for measuring motion was established with a mechanical device that moved the unit back and forth through a consistent arc of movement. The unit was tested three times, each time for 5 minutes. The mean vector magnitudes (MVMs) of each these three tests were 776,688 , and 772 au, respectively ( mean \pm standard deviation $=745.3 \pm 49.7 \mathrm{au}$ ).

\section{Mobility Tasks}

The three mobility tasks were-

1. A 5-minute walk test, in which participants walked along a flat designated route at their natural comfortable walking speed. The distance walked in 5 minutes was recorded in meters. We chose the 5-minute duration based on a previous validation study of the TriTrac R3D accelerometer (Stayhealthy, Inc), which included participants with MS with similar disability ratings as the MS participants in this study.

2. The timed up and go test [25], defined as the time to rise from sitting, walk $3 \mathrm{~m}$ at a natural comfortable walking speed, turn, walk back $3 \mathrm{~m}$, and sit back down.

3. A stair-climbing task, in which participants climbed up 10 steps (measuring $18 \mathrm{~cm}$ in height), with or without

\footnotetext{
*Mulcare JA, Mathews T. Unpublished observations, 2004.
}

the use of the rails and/or assistive devices (the assistance required was noted and participants were instructed to use the same assistance during the second test). The time taken to climb the 10 steps was recorded.

Tasks were performed in the same order on all occasions. A 2-minute rest period, sitting in a chair, was interspersed between each task, and participants rested for 5 minutes before and after completing all three tasks.

\section{Data and Statistical Analysis}

The acceleration data (meters per second squared) measured during each mobility task by the RT3 for each of its three axes were computed by the RT3 Assist software (Stayhealthy, Inc) into the MVM (MVM $=\left(x^{2}+y^{2}+\right.$ $\left.\left.z^{2}\right) \times 0.5\right)$. MVM data were expressed in au. All statistical analyses were performed using the SPSS 12.0 Software for Windows (SPSS Inc, Chicago, Illinois). All demographic and measured data were analyzed descriptively. Independent $t$-tests were used to compare differences between group with MS and control group mean weight and age.

We analyzed the reliability of the accelerometer measurements using the MVM for each of the three mobility tasks on each of the 2 test days. We used ICCs (ICC, 1:1) with a one-way random model and the standard error of measurement (SEM) as follows [26]: ICC = between-subject variance/(between-subject variance + within-subject variance), and SEM = square root of the within-subject variance.

Correlation strength was determined as follows: 0.00 to 0.25 = little, if no correlation; 0.26 to $0.49=$ low correlation; 0.50 to $0.69=$ moderate correlation; 0.70 to $0.89=$ high correlation; and 0.90 to 1.00 = very high correlation [27].

\section{RESULTS}

\section{Participants}

Ten participants with MS (nine females and one male) aged 35 to $60 \mathrm{yr}$ (mean $49 \mathrm{yr}$ ), with a wide range of mobility levels and EDSS scores ranging from 1.5-6.0 volunteered for the study. The duration of MS varied from 2 to $37 \mathrm{yr}$. Seven participants had relapsing-remitting MS, and three participants were diagnosed with the slowly progressive form of the disease. Mobility ranged from two participants who were able to walk without detectable disability to two participants who required two crutches 
with which to walk and one participant who walked with a walker (Table 1). The 10 control participants (7 females and 3 males) were significantly younger (33 to $48 \mathrm{yr}$, mean $40 \mathrm{yr}, p<0.05$ ) than the participants with MS. No significant difference was found between the two groups with regard to weight $(p<0.05)$ (Table 2). Descriptive MVM and mobility-task data collected from MS and control participants are shown in Tables 3 and 4, respectively.

\section{Test-Retest Reliability}

The accelerometry data (presented as MVM in au) demonstrated a moderate test-retest reliability over 1 week for the participants with MS for the 5-minute walk test (ICC $=0.64, p=0.01$ ) and the timed up and go test (ICC $=0.50, p=0.05$ ). A higher correlation was found for the stair-climbing task (ICC $=0.76, p=0.002$ ) (Table 5). Although the ICC values were statistically significant, the confidence interval (CI) and SEM were large, reflecting the small sample size.

The ICC of the accelerometry data for the control participants was similar to that of the MS participants for the 5-minute walk test (ICC $=0.65$ ), but the ICC values for the timed up and go test (ICC $=-0.04$ ) and the stairclimbing task (ICC $=0.39$ ) were extremely low (Table 5).

Table 6 reflects the test-retest reliability analysis of the distance data for the 5-minute walk test and the time data for the timed up and go test and stair-climbing task for both the MS and the control participants. The ICCs for these tasks were high, ranging from 0.74 to 0.96 ( $p<$ 0.05 ) across all three tasks in both groups.

Table 1.

Demographic data for participants with multiple sclerosis (MS) $(n=10)$.

\begin{tabular}{|c|c|c|c|c|c|}
\hline Participant & Type of MS* & $\begin{array}{c}\text { Duration } \\
\text { of MS (yr) }\end{array}$ & $\begin{array}{c}\text { Severity Level } \\
\left.\text { (EDSS }{ }^{*} \text { score } / 10\right)\end{array}$ & $\begin{array}{l}\text { Walking Ability Level } \\
\text { (RMI score/15) }\end{array}$ & $\begin{array}{c}\text { Assistive Devices } \\
\text { Required for Walking }\end{array}$ \\
\hline 1 & R-R & 12 & 3.5 & 12 & 1 Elbow crutch \\
\hline 2 & Mild & 4 & 1.5 & 15 & None \\
\hline 3 & $\mathrm{R}-\mathrm{R}$ & 20 & 3.5 & 14 & AFO \\
\hline 4 & $\mathrm{R}-\mathrm{R}$ & 37 & 3.0 & 15 & None \\
\hline 5 & $\mathrm{R}-\mathrm{R}$ & 28 & 3.5 & 13 & 1 Elbow crutch \\
\hline 7 & SP & 24 & 6.0 & 11 & 2 Elbow crutches \\
\hline 8 & $\mathrm{R}-\mathrm{R}$ & 2 & 2.0 & 15 & None \\
\hline 9 & SP & 5 & 5.0 & 13 & 2 Elbow crutches \\
\hline 10 & R-R & 25 & 2.5 & 14 & 1 Elbow crutch \\
\hline Range & - & $2-37$ & $1.5-6.0$ & $11-15$ & - \\
\hline
\end{tabular}

Table 2.

Comparison of demographic data for group with multiple sclerosis (MS) and control group.

\begin{tabular}{|c|c|c|c|c|c|c|}
\hline \multirow{2}{*}{ Variable } & \multicolumn{2}{|c|}{ MS } & \multicolumn{2}{|c|}{ Control } & \multirow{2}{*}{$t$-Value } & \multirow{2}{*}{$p$-Value } \\
\hline & Mean \pm SD & Range & Mean \pm SD & Range & & \\
\hline Height (cm) & $160 \pm 6$ & 152-169 & $173 \pm 9$ & 164-190 & 3.1 & $0.01^{*}$ \\
\hline Weight (kg) & $71 \pm 17$ & 49-98 & $74 \pm 14$ & $52-101$ & 0.30 & 0.77 \\
\hline Age (yr) & $49 \pm 9$ & $35-60$ & $40 \pm 6$ & 33-48 & -3.2 & $0.01^{*}$ \\
\hline Female $(n)$ & \multicolumn{2}{|c|}{9} & \multicolumn{2}{|c|}{7} & - & - \\
\hline Male (n) & \multicolumn{2}{|c|}{1} & \multicolumn{2}{|c|}{3} & - & - \\
\hline
\end{tabular}


Table 3.

Multiple sclerosis (MS) participant data from 5-minute walk test (mean vector magnitude [MVM] in activity units [au], distance in meters), timed up and go test (MVM, time in seconds), and stair-climbing task (MVM, time in seconds) ( $n=10)$ on two occasions separated by 1 week.

\begin{tabular}{|c|c|c|c|c|c|c|c|c|c|c|c|c|}
\hline \multirow[b]{2}{*}{ MS } & \multicolumn{4}{|c|}{ 5-Minute Walk Test } & \multicolumn{4}{|c|}{ Timed Up and Go Test } & \multicolumn{4}{|c|}{ Stair-Climbing Task } \\
\hline & \multicolumn{2}{|c|}{ MVM (au) } & \multicolumn{2}{|c|}{ Distance (m) } & \multicolumn{2}{|c|}{ MVM (au) } & \multicolumn{2}{|c|}{ Time (s) } & \multicolumn{2}{|c|}{ MVM (au) } & \multicolumn{2}{|c|}{ Time (s) } \\
\hline 1 & 3,575 & 10,847 & 192 & 224 & 186 & 330 & 12.2 & 12.3 & 141 & 201 & 13.4 & 11.9 \\
\hline 3 & 8,180 & 18,649 & 236 & 249 & 352 & 882 & 15.2 & 13.4 & 145 & 239 & 21.4 & 19.2 \\
\hline 4 & 5,486 & 20,770 & 223 & 275 & 257 & 420 & 12.5 & 11.7 & 173 & 205 & 13.0 & 11.9 \\
\hline 5 & 23,673 & 26,111 & 140 & 110 & 619 & 1,165 & 14.0 & 12.2 & 345 & 295 & 15.8 & 14.1 \\
\hline 8 & 19,744 & 11,817 & 319 & 316 & 382 & 323 & 9.0 & 9.4 & 179 & 124 & 9.8 & 10.3 \\
\hline 9 & 931 & 1,895 & 46 & 43 & 156 & 369 & 49.0 & 45.0 & 354 & 458 & 60.0 & 75.0 \\
\hline 10 & 2,726 & 4,554 & 140 & 206 & 161 & 193 & 20.6 & 11.1 & 82 & 182 & 29.2 & 33.5 \\
\hline$\overline{\text { Mean }}$ & 7,778 & 10,933 & 197 & 211 & 272 & 441 & 17.5 & 16.0 & 197 & 228 & 21.9 & 23.3 \\
\hline SD & 7,633 & 8,336 & 83 & 88 & 145 & 325 & 11.6 & 10.6 & 89 & 97 & 15.4 & 20.2 \\
\hline
\end{tabular}

Table 4.

Control participant data from 5-minute walk test (mean vector magnitude [MVM] in activity units [au], distance in meters), timed up and go test (MVM, time in seconds), and stair-climbing task (MVM, time in seconds) ( $n=10)$ on two occasions separated by 1 week.

\begin{tabular}{|c|c|c|c|c|c|c|c|c|c|c|c|c|}
\hline \multirow[b]{2}{*}{ Control } & \multicolumn{4}{|c|}{ 5-Minute Walk Test } & \multicolumn{4}{|c|}{ Timed Get Up and Go Test } & \multicolumn{4}{|c|}{ Stair-Climbing Task } \\
\hline & \multicolumn{2}{|c|}{ MVM (au) } & \multicolumn{2}{|c|}{ Distance (m) } & \multicolumn{2}{|c|}{ MVM (au) } & \multicolumn{2}{|c|}{ Time (s) } & \multicolumn{2}{|c|}{ MVM (au) } & \multicolumn{2}{|c|}{ Time (s) } \\
\hline 1 & 5,847 & 4,237 & 365 & 349 & 435 & 58 & 7.5 & 6.9 & 154 & 102 & 7.5 & 7.6 \\
\hline 3 & 11,671 & 9,580 & 354 & 355 & 360 & 277 & 8.4 & 8.0 & 266 & 204 & 7.6 & 7.4 \\
\hline 4 & 7,071 & 10,465 & 358 & 346 & 202 & 154 & 8.7 & 8.5 & 158 & 222 & 9.3 & 9.6 \\
\hline 7 & 8,573 & 12,000 & 299 & 220 & 129 & 390 & 9.3 & 9.8 & 116 & 174 & 9.0 & 9.1 \\
\hline 8 & 7,474 & 8,369 & 361 & 376 & 120 & 169 & 6.9 & 7.3 & 224 & 214 & 7.3 & 7.9 \\
\hline 9 & 10,330 & 9,944 & 356 & 369 & 394 & 261 & 8.0 & 8.6 & 182 & 191 & 8.5 & 8.8 \\
\hline 10 & 5,673 & 6,724 & 403 & 420 & 140 & 131 & 7.6 & 7.2 & 181 & 165 & 8.0 & 7.8 \\
\hline$\overline{\text { Mean }}$ & 7,932 & 8,543 & 361 & 361 & 237 & 189 & 8.0 & 8.0 & 200 & 177 & 8.3 & 8.3 \\
\hline
\end{tabular}

\section{DISCUSSION}

Motion data collected from an accelerometer can potentially be used to determine the amount of activity a person engages in from day to day or measure discrete motor tasks. Therefore, accelerometers could, theoretically, be used to measure the effectiveness of physical therapy intervention; however, the measurement properties of the RT3 must first be established.

Few studies have investigated the ability of the RT3 to produce stable data on repeated measurements. The reli- ability of the RT3 has been studied with a mechanical motorized vibration table, which was assessed with a strain-gauge accelerometer and found to produce consistent movement [13]. A low intramonitor variation $(<1.3 \pm$ $0.9 \%$ mean coefficient of variation at $10.2 \mathrm{~Hz}$ vibration frequency) and a high intermonitor reliability (ICC $=0.99$, $p<0.001$ ) of the RT3 were demonstrated [13]. In a further reliability study, a person without disability performed six activities on two occasions separated by 2 days [14]. Eight RT3 monitors were used to measure motion, and no significant differences $(p<0.001)$ were found for data 
Table 5.

Results of test-retest reliability analysis (intraclass correlation coefficient [ICC] and standard error of measurement [SEM]) of mean vector magnitude (MVM) data in activity units and MVM data normalized by mobility data collected on test day 1 compared with test day 2 for all three mobility tasks for participants with multiple sclerosis (MS) $(n=10)$ versus control $(n=10)$ participants.

\begin{tabular}{|c|c|c|c|c|c|c|}
\hline \multirow{2}{*}{ Test } & \multicolumn{2}{|c|}{ ICC (95\% CI) } & \multicolumn{2}{|c|}{$p$-Value* } & \multicolumn{2}{|c|}{ SEM (\% difference) } \\
\hline & MS & Control & MS & Control & MS & Control \\
\hline \multicolumn{7}{|l|}{$\overline{\text { 5-Minute Walk }}$} \\
\hline MVM & 0.64 (0.09 to 0.89$)$ & 0.65 (0.11 to 0.90$)$ & 0.01 & 0.01 & $4,862(63)$ & $1,296(16)$ \\
\hline Normalized (MVM/m) & 0.83 (0.50 to 0.96$)$ & 0.55 (-0.05 to 0.86$)$ & 0.001 & 0.04 & $23.5(57)$ & $6.5(29)$ \\
\hline \multicolumn{7}{|l|}{ Timed Up and Go } \\
\hline MVM & $0.50(-0.11$ to 0.85$)$ & -0.04 (-0.61 to 0.57$)$ & 0.05 & 0.54 & $186(68)$ & $112(47)$ \\
\hline Normalized (MVM/s) & $0.50(-0.12$ to 0.84$)$ & $-0.08(-0.63$ to 0.54$)$ & 0.05 & 0.59 & $16.0(80)$ & $15.3(51)$ \\
\hline \multicolumn{7}{|l|}{ Stair Climbing } \\
\hline MVM & 0.76 (0.32 to 0.93$)$ & 0.39 (-0.25 to 0.80$)$ & 0.002 & 0.11 & $46(23)$ & $42(21)$ \\
\hline Normalized (MVM/s) & 0.74 (0.28 to 0.93$)$ & 0.55 ( -0.05 to 0.86$)$ & 0.01 & 0.04 & $2.8(26)$ & $4.6(17)$ \\
\hline
\end{tabular}

Table 6.

Results of test-retest reliability analysis (intraclass correlation coefficient [ICC] and standard error of measurement [SEM]) for distance data of 5-minute walk test and time data from timed up and go test and stair-climbing task collected on test day 1 compared with test day 2 for participants with multiple sclerosis (MS) $(n=10)$ versus control $(n=10)$ participants.

\begin{tabular}{|c|c|c|c|c|c|c|}
\hline \multirow{2}{*}{ Test } & \multicolumn{2}{|c|}{ ICC (95\% CI) } & \multicolumn{2}{|c|}{$p$-Value* } & \multicolumn{2}{|c|}{ SEM (\% difference) } \\
\hline & MS & Control & MS & Control & MS & Control \\
\hline 5-Minute Walk (m) & $0.94(0.78$ to 0.98$)$ & $0.74(0.28$ to 0.93$)$ & 0.001 & 0.003 & $20.3(10)$ & $22.1(6)$ \\
\hline Timed Up and Go (s) & $0.95(0.83$ to 0.99$)$ & $0.79(0.39$ to 0.94$)$ & 0.001 & 0.001 & 3.3 (19) & $0.36(5)$ \\
\hline
\end{tabular}

${ }^{*}$ Significance set at $p=0.05$.

CI = confidence interval.

collected on each occasion [14]. The measurement properties of the RT3 for physical activity in people with disability have not, to our knowledge, been investigated. This pilot study investigated the test-retest reliability of motion data measured by a single RT3 during the performance of three discrete mobility tasks in a small sample of adults with MS compared with a control sample.

We found that the RT3 data for the 5-minute walk, the timed up and go, and the stair-climbing mobility tasks for the participants with MS were only moderately reliable (ICC $=0.64,0.50$, and 0.76, respectively; $p<0.05$ ) (Table 5). This result could be interpreted in two ways. Either the RT3 monitor was not consistent in its measurement of motion or the participants' performance varied slightly from test day to test day, in spite of our attempts to standardize the tests and conditions. The RT3 used in this study yielded consistent measurements when tested mechanically; however, even though the placement of the unit on each participant was standardized to the middle of the lower back, slight variations in placement and unit movement on the body may have resulted in measurement variation. In the reliability study conducted on one nondisabled adult, the authors acknowledged that individual RT3 unit movement on the participant at the site of attachment may have accounted for some of the variation seen in their data, even though they had ensured that the RT3 monitors were firmly taped together in packs of four and attached to a tight belt around the participant's waist [14]. In the present study, we placed the RT3 unit in the supplied clip, which was then attached to the belt of the participant's trousers or skirt per the manufacturer's instructions. This pragmatic, though less rigid method of attachment, mimics that which would be used in freeliving studies, where participants would be responsible for attaching the unit; however, it may have allowed movement of the RT3 unit against the body, which was exacerbated when the participants performed the timed up and go test and the stair-climbing task.

We also know that the symptoms of MS can vary from day to day, potentially altering motor performance 
$[4,28]$; however, the distance and time data collected from the participants with MS for the 5-minute walk, the timed up and go, and the stair-climbing task in this study demonstrated a very high correlation between the two test days (ICC $=0.94,0.95$, and 0.96, respectively; $p<0.001$ ) (Table 6). We can see in Tables 3 and 4 that participants did not walk the same distance on both 5-minute walk tests nor did they take the same time to complete the other two mobility tasks, which may have resulted in corresponding variations in the RT3 data. To account for this, we divided the RT3 data by the data from each of the mobility tasks (distance and time) in turn (Table 5). This only slightly improved the ICC values for the 5-minute walk test and did not alter the ICC values for the other two mobility tasks.

The control RT3 data demonstrated moderate reliability for the 5-minute walk test only (ICC $=0.65$; $p<$ 0.01) (Table 5); the stability of the RT3 data for the other two mobility tasks was poor (ICC $=-0.04,0.39 ; p<0.54$, for timed up and go and stair climbing, respectively) (Table 5). The control group distance and time data for the three mobility tasks was found to be highly correlated but not as high as for the participants with MS (ICC = $0.74,0.79,0.87 ; p<0.003$, for 5-minute walk, timed up and go, and stair climbing, respectively) (Table 6), which indicates that control participants were varying slightly the nature of their motor performances from day to day, possibly exacerbating the attachment motion of the RT3. Normalizing the RT3 data for the control participants by dividing these data by the mobility-task data did not substantially alter the ICC values (Table 5).

The control group RT3 data for the mobility tasks were more variable than the RT3 data for the participants with MS. A possible explanation for this finding may be that motor performance for a specific motor task in the absence of disability may be more variable than it is in the presence of disability. The participants with MS, in spite of the changeable nature of MS symptoms, may therefore move with less variable movement patterns than their nondisabled counterparts. Because the motor tasks were short and participants were able to rest before beginning each task, fatigue may not have been an important factor for the participants with MS. The use of motor planning could be another possible explanation of the performance variations between the MS and control participants. The tasks in this study were easy everyday tasks for the control participants, who executed the movements automatically with little motor planning, which may have resulted in performance variations. However, for people with a disability, the tasks may have presented a greater challenge, requiring motor planning to execute and thus resulting in a more static performance. In addition, a significant difference existed in age between the group with MS and control group; the latter group was approximately a decade older. Perhaps older adult movement behavior is less variable than that of younger adults, and we recommend that future research compare the variability of younger and older control participants.

The results of this small sample size pilot study indicate that (1) the RT3 provides moderately stable data when measuring walking and (2) the variation in the motion data in the present study may be largely explained by the method of attachment of the RT3 to the participant and, to a lesser extent, by changes in participant performance. These results warrant further investigation with a larger sample as well as development of a more robust method of attachment than suggested by the manufacturer. The latter issue could pose a problem when the RT3 is used in free-living studies because a simple participant-friendly attachment method would be required. The changes in participant motor performance on repeated testing reported in the present study present an additional source of variability that would need to be addressed when similar studies are designed.

Data from this pilot study were used in a sample size calculation described by Bonett [29], the results of which indicated that to investigate test-retest reliability with one repeat measurement, researchers would require 53 participants to obtain an ICC value with a 95 percent CI of width 0.2. More studies with larger samples are also required to evaluate whether the RT3 can provide stable data on repeated measurements of short motor tasks, other than walking, in both nondisabled and disabled groups, as well as for longer periods, such as when measuring daily physical activity. The RT3 may be better suited to measuring activity over longer periods than short discrete motor tasks.

\section{CONCLUSIONS}

This article reports on the first study of the latest and, allegedly, more sensitive model of the triaxial accelerometers, the RT3, in a sample of adults with MS and nondisabled adults. Test-rest reliability of data collected 1 week 
apart at the same time of day with the RT3 accelerometer was moderate in people with MS and nondisabled adults for the 5-minute walk test, poor in both groups for the timed up and go test, and moderately high for stair climbing in the group with MS. We suggest that variability in measurement might be due to two sources of measurement error. The pragmatic attachment method used in the present study may have allowed movement of the RT3 at the site of attachment. In addition, both participant groups demonstrated inconsistencies in the performance of repeated standardized motor tasks.

We found that the RT3 appears to reliably measure walking and thus has the potential to be a mobility outcome measure both in adults with MS and in nondisabled adults. Further studies to verify these findings, with larger sample sizes and more robust RT3 attachment methods, are justified and should also include a range of motor tasks as well as daily physical activity. Based on the results of the 5-minute walk test in this study, a sample of 53 participants would be required to obtain an ICC value with a 95 percent $C I$ of width 0.2 from two repeat measurements.

\section{ACKNOWLEDGMENTS}

We would like to thank all the participants; Robyn Bell (School of Physical Education, University of Otago, Dunedin, New Zealand) and Jan Piggot (School of Physiotherapy, University of Otago) for technical advice; and Dr. Melanie Bell (Department of Preventive and Social Medicine, University of Otago) for statistical advice.

This material is the result of work partly supported by a scholarship for a Summer Studentship from the Health Research Council of New Zealand.

The authors have declared that no competing interests exist.

\section{REFERENCES}

1. Ng AV, Kent-Braun JA. Quantification of lower physical activity in persons with multiple sclerosis. Med Sci Sports Exerc. 1997;29(4):517-23. [PMID: 9107635]

2. Petajan JH, Gappmaier E, White AT, Spencer MK, Mino L, Hicks RW. Impact of aerobic training on fitness and quality of life in multiple sclerosis. Ann Neurol. 1996;39(4):432-41. [PMID: 8619521]
3. Ponichtera-Mulcare JA, Mathews T, Barrett PJ, Grupta SC. Change in aerobic fitness of patients with multiple sclerosis during 6-month training program. Sports Med Train Rehabil. 1997;7:265-72.

4. White LJ, Dressendorfer RH. Exercise and multiple sclerosis. Sports Med. 2004;34(15):1077-1100. [PMID: 15575796]

5. Westerterp KR. Physical activity assessment with accelerometers. Int J Obes Relat Metab Disord. 1999;23 Suppl 3:S45-49. [PMID: 10368002]

6. Bassett DR, Cureton AL, Ainsworth BE. Measurement of daily walking distance-questionnaire versus pedometer. Med Sci Sports Exerc. 2000;32(5):1018-23. [PMID: 10795795]

7. Mathie MJ, Coster AC, Lovell NH, Celler BG. Accelerometry: Providing an integrated, practical method for longterm, ambulatory monitoring of human movement. Physiol Meas. 2004;25(2):R1-20. [PMID: 15132305]

8. Melanson EL, Knoll JR, Bell ML, Donahoo WT, Hill JO, Nysse LJ, Lanningham-Foster L, Peters JC, Levine JA. Commercially available pedometers: Considerations for accurate step counting. Prev Med. 2004;39(2):361-68. [PMID: 15226047]

9. Rowlands AV, Thomas PW, Eston RG, Topping R. Validation of the RT3 triaxial accelerometer for the assessment of physical activity. Med Sci Sports Exerc. 2004;36(3):518-24. [PMID: 15076796]

10. King GA, Torres N, Potter C, Brooks TJ, Coleman KJ. Comparison of activity monitors to estimate energy cost of treadmill exercise. Med Sci Sports Exerc. 2004;36(7): 1244-51. [PMID: 15235333]

11. DeVoe D, Gotshall R, McArthur T. Comparison of the RT3 Research Tracker and Tritrac R3D accelerometers. Percept Mot Skills. 2003;97(2):510-18. [PMID: 14620239]

12. Kozub FM, Oh H-K, Rider RA. RT3 accelerometer accuracy in estimating short term physical activity in individuals with visual impairments. Adapt Phys Activ Q. 2005; 22(3):265-76.

13. Powell SM, Jones DI, Rowlands AV. Technical variability of the RT3 accelerometer. Med Sci Sports Exerc. 2003; 35(10):1773-78. [PMID: 14523319]

14. Powell SM, Rowlands AV. Intermonitor variability of the RT3 accelerometer during typical physical activities. Med Sci Sports Exerc. 2004;36(2):324-30. [PMID: 14767258]

15. Welk GJ, Schaben JA, Morrow JR. Reliability of accelerometry-based activity monitors: A generalizability study. Med Sci Sports Exerc. 2004;36(9):1637-45. [PMID: 15354049]

16. Coleman KJ, Saelens BE, Wiedrich-Smith MD, Finn JD, Epstein LH. Relationships between TriTrac-R3D vectors, heart rate, and self-report in obese children. Med Sci Sports Exerc. 1997;29(11):1535-42. [PMID: 9372493]

17. Leenders JM, Sherman WM, Nagaraja HN, Kien CL. Evaluation of methods to assess physical activity in free-living 
conditions. Med Sci Sports Exerc. 2001;33(7):1233-40. [PMID: 11445774$]$

18. Jakicic JM, Winters C, Lagally K, Ho J, Robertson RJ, Wing RR. The accuracy of the TriTrac-R3D accelerometer to estimate energy expenditure. Med Sci Sports Exerc. 1999; 31(5):747-54. [PMID: 10331898]

19. Welk GJ, Blaire SN, Wood K, Jones S, Thompson RW. A comparative evaluation of three accelerometry-based physical activity monitors. Med Sci Sports Exerc. 2000; 32(9 Suppl):S489-97. [PMID: 10993419]

20. Welk GJ, Corbin CB. The validity of the TriTrac-R3D Activity Monitor for the assessment of physical activity in children. Res Q Exerc Sport. 1995;66(3):202-9. [PMID: 7481081]

21. Nichols JF, Morgan CG, Sarkin JA, Sallis JF, Calfas KJ. Validity, reliability, and calibration of the Tritrac accelerometer as a measure of physical activity. Med Sci Sports Exerc. 1999;31(6):908-12. [PMID: 10378921]

22. Poser CM, Paty DW, Scheinberg L, McDonald WI, Davis FA, Eberts GC, Johnson KP, Sibley WA, Silberberg DH, Tourtellotte WW. New diagnostic criteria for multiple sclerosis: Guidelines for research protocols. Ann Neurol. 1983; 13(3):227-31. [PMID: 6847134]
23. Kurtzke JF. Rating neurological impairment in multiple sclerosis: An Expanded Disability Status Scale (EDSS). Neurology. 1983;33(11):1444-52. [PMID: 6685237]

24. Collen FM, Wade DT, Robb GF, Bradshaw CM. The Rivermead Mobility Index: A further development of the Rivermead Motor Assessment. Int Disabil Stud. 1991;13(2):50-54. [PMID: 1836787

25. Podsiadlo D, Richardson S. The timed "Up \& Go": A test of basic functional mobility for frail elderly persons. J Am Geriatr Soc. 1991;39(2):142-48. [PMID: 1991946]

26. Finch E, Canadian Physiotherapy Association. Physical rehabilitation outcome measures: A guide to enhanced clinical decision making. Hamilton (Ontario): BC Decker; 2002.

27. Domholdt E. Physical therapy research: Principles and applications. 2nd ed. Philadelphia (PA): W.B. Saunders; 2000.

28. Thompson AJ. Symptomatic management and rehabilitation in multiple sclerosis. J Neurol Neurosurg Pyschiatry. 2001;71 Suppl 2:ii22-27. [PMID: 11701781]

29. Bonett DG. Sample size requirements for estimating intraclass correlations with desired precision. Stat Med. 2002;21(9): 1331-35. [PMID: 12111881]

Submitted for publication September 22, 2005. Accepted in revised form April 23, 2007. 